\title{
The Underlying Role of Mitophagy in Different Regulatory Mechanisms of Chronic Obstructive Pulmonary Disease
}

This article was published in the following Dove Press journal: International Journal of Chronic Obstructive Pulmonary Disease

\author{
Jian-Yu Liu (D)' \\ Meng-Yu Zhang' \\ Yi-Qing Qu (iD ${ }^{2}$ \\ 'Department of Pulmonary and Critical \\ Care Medicine, Qilu Hospital, Cheeloo \\ College of Medicine, Shandong \\ University, Jinan, Shandong, People's \\ Republic of China; ${ }^{2}$ Department of \\ Pulmonary and Critical Care Medicine, \\ Qilu Hospital of Shandong University, \\ Jinan, Shandong, People's Republic of \\ China
}

\begin{abstract}
COPD is a common disease of the respiratory system. Inflammation, cellular senescence and necroptosis are all pathological alterations of this disease, which may lead to emphysema and infection that aggravate disease progression. Mitochondria acting as respiration-related organelles is usually observed with abnormal changes in morphology and function in CS-stimulated models and COPD patients. Damaged mitochondria can activate mitophagy, a vital mechanism for mitochondrial quality control, whereas under the persistent stimulus of CS or other forms of oxidative stress, mitophagy is impaired, resulting in insufficient clearance of damaged mitochondria. However, the excessive activation of mitophagy also seems to disturb the pathology of COPD. In this review, we demonstrate the variations in mitochondria and mitophagy in CS-induced models and COPD patients and discuss the underlying regulatory mechanism of mitophagy and COPD, including the roles of inflammation, senescence, emphysema and infection.
\end{abstract}

Keywords: mitophagy, COPD, different regulatory mechanisms

\section{Introduction}

Chronic obstructive pulmonary disease (COPD) contributes significantly to the global burden of disease as the fourth leading cause of mortality worldwide. ${ }^{1}$ COPD is characterized by persistent symptoms of the respiratory system, and progressive airway limitation is principally caused by the harmful effects of cigarette smoke (CS) exposure. ${ }^{2}$ Recent studies have shown that CS can cause cellular senescence by generating excessive reactive oxygen species (ROS) in mitochondria impair mitochondrial functions, including mitophagy. ${ }^{3,4}$ Mitochondria are not only primary endogenous producers of ROS but also major targets of ROS, which result in abnormal inflammatory responses under conditions of persistent and high levels of mitochondrial ROS (mtROS). ${ }^{5}$ Researchers suggest that the accumulation of mtROS caused by CS activates inflammatory pathways by interfering with mitochondrial quality control. ${ }^{6,7}$ The stressed mitochondria fuse together and then the damaged contents and healthy mitochondria are separated by a fission mechanism, ${ }^{8}$ and then, the impaired mitochondria are mainly degraded via mitophagy ${ }^{9}$ to control and maintain mitochondrial quality. The mitochondrial morphology in primary bronchial epithelial cells from COPD patients is altered, presenting with swollen, elongated and fragmented mitochondria with loss of cristae. ${ }^{10}$ In alveolar epithelial cells, a mild level of CS can induce mitochondrial hyperfusion and render these
Correspondence: Yi-Qing Qu

Department of Pulmonary and Critical

Care Medicine, Qilu Hospital of Shandong

University, Jinan, Shandong, People's

Republic of China

$\mathrm{Tel}+8653182169335$

Fax +8653182967544

Email quyiqing@sdu.edu.cn
International Journal of Chronic Obstructive Pulmonary Disease 2020:15 2167-2I77 
cells more sensitive to additional stress. ${ }^{11}$ Mitochondrial hyperfusion and fragmentation are both adaptive measures of mitochondrial quality control. These alterations are partly attributed to impaired mitophagy caused by CS exposure, which induces a deteriorating mitochondrial state. A recent study demonstrated that CS impairs mitophagy, which results in the accumulation of damaged mitochondria., ${ }^{3,12}$ Researchers have also found that damaged mitochondria accumulate in the perinuclear region and generate excessive mtROS, which give rise to nuclear DNA damage and contribute to subsequent cellular senescence. ${ }^{12}$ In addition, a series of pathological changes in the innate immune system resulting from the impairment of mitophagy also plays a nonnegligible role in the pathogenesis of COPD. ${ }^{13,14-16}$ However, the upregulated level of mitophagy has also been shown to cause mitophagy-dependent necroptosis, which is correlated with emphysema in COPD. ${ }^{17}$ This review summarizes the different pathological alterations caused by COPD when mitophagy is damaged by the persistent activation by CS (Figure 1).

\section{The Alterations of Mitochondria and Mitophagy During CS Exposure or in COPD}

Mitochondria are double-membrane organelles that have preserved and specific structures that are mainly regulated by genomic DNA while taking part in the functioning of integrated intracellular systems. ROS, mainly released from mitochondria, act as second messengers to maintain cellular homeostasis under physiological conditions. ${ }^{18}$

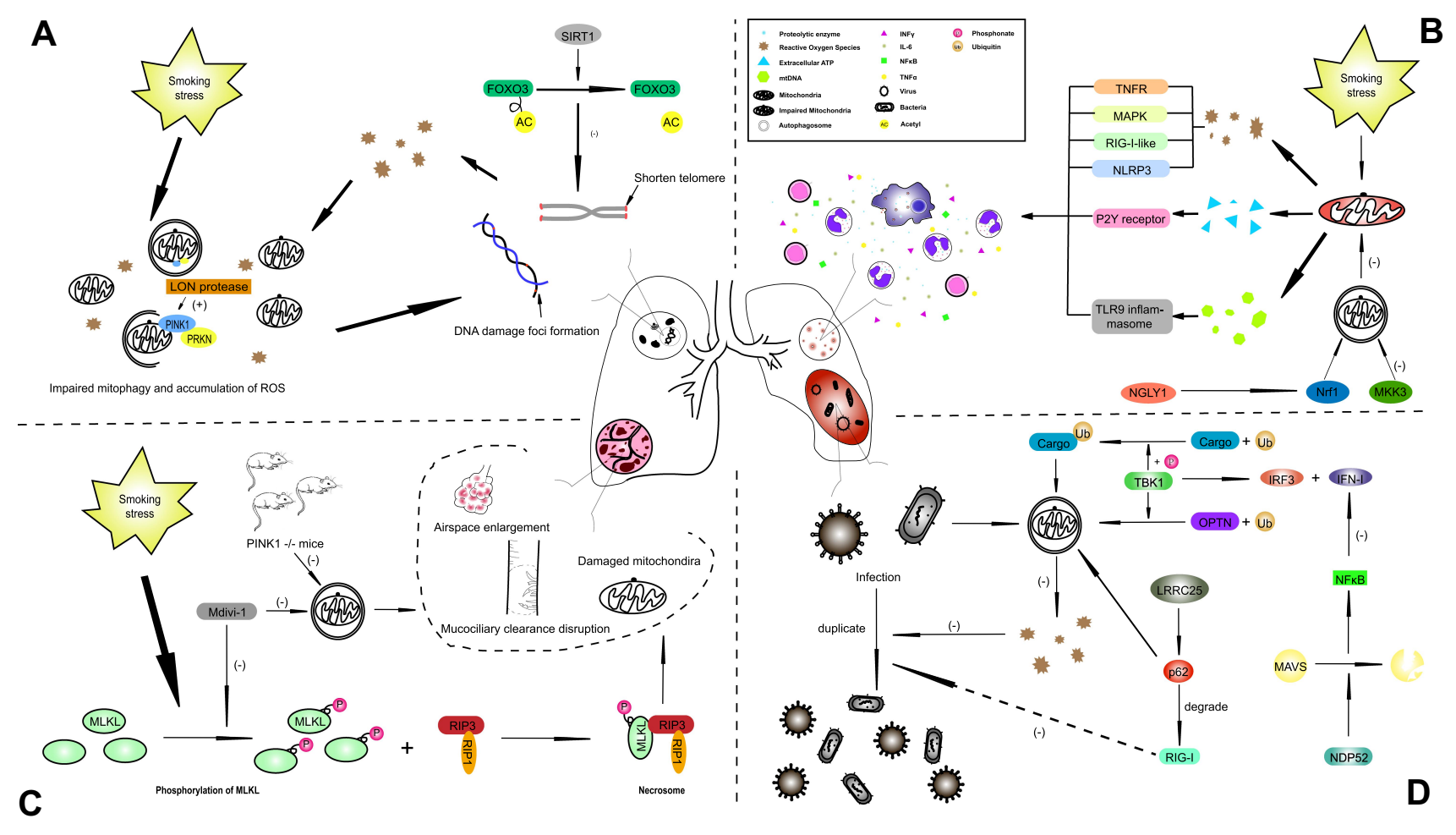

Figure I The underlying mechanism of mitophagy in influencing COPD with different phenotypes. (A) The stimulus of smoking-induced stress induces excessive generation of ROS, injuries to mitochondria and impaired mitophagy, and these ROS target DNA, leading to DNA damage foci formation, which causes cellular senescence and establishes a vicious cycle. (B) In the lung tissue of COPD patients, several inflammatory cells, such as macrophages, neutrophils and CD8+ T cells, and cytokines were observed, including IFN $\gamma$, IL-6, NFKB and TNF $\alpha$. Insufficient mitophagy cannot eliminate damaged mitochondria efficiently, which leads to the generation of excessive ROS, ATP and proteolytic enzymes. (C) Emphysema is caused by the basic pathological changes of COPD. CS-induced necroptosis contributes to the alteration observed in emphysema, and the phosphorylation of MLKL plays a precursor role in necroptosis-related emphysema, which can be attenuated by Mdivi-I and PINKI knockout. (D) Infections with bacteria or viruses can also act on molecular targets of mitophagy under conditions of mitophagy dysfunction. ROS constitute a defensive mechanism for pathogens, and overstimulated mitophagy can weaken this protective effect of ROS. The innate immune system is also affected by mitophagy through many signaling pathways, such as the TBKI pathway.

Abbreviations: AC, acetyl; COPD, Chronic obstructive pulmonary disease; CS, cigarette smoke; FOXO3, forkhead box O3; IL, interleukin; LRRC25, leucine-rich repeat containing protein 25; MAPK, mitogen activated protein kinase; MAVS, mitochondrial antiviral-signaling protein; MKK3, mitogen activated protein kinase kinase 3; MLKL, mixed lineage kinase domain like pseudokinase; mtDNA, mitochondrial DNA; NDP52, calcium binding and coiled-coil domain 2; NFKB, nuclear factor kappa B; NGLYI, $\mathrm{N}$-glycanase I; NLRP3, NLR family pyrin domain containing 3; NRFI, nuclear factor, erythroid 2 like I; OPTN, optineurin; PINKI, PTEN-induced putative kinase I; PRKN, parkin RBR E3 ubiquitin protein ligase; RIP3, receptor-interacting serine-threonine kinase 3; RLR, RIG-I like receptor; ROS, reactive oxygen species; SIRT, sirtuin; TBKI, TANK binding kinase I; TLR9, toll like receptor 9; TNFR, tumor necrosis factor receptor. 
Mitochondrial DNA (mtDNA) is sensitive to oxidant stress, ${ }^{19}$ and the oxidization of mtDNA accelerates the aging process, resulting in accumulating mutations in the mitochondrial genome, which reduces the efficiency of electron transport chain activity and increases ROS generation. ${ }^{20}$ Persistent stimulation of CS causes excessive ROS generation by damaged mitochondria, which promotes a critical and propagating cellular stress response. ${ }^{21}$ Impaired mitochondria can also be the prime targets of ROS, leading to further impairment of mitochondrial proteins and mtDNA and hence creating a vicious circle in the mitochondrial network. The mitochondrial network is a dynamic structure that adapts to attenuate various environmental stresses through the fusion and fission of mitochondria and exchange of mtDNA, ATP and other components to maximize the efficiency of energy production. ${ }^{11,22}$ The homeostasis of mitochondrial fission and fusion can ensure a sufficient number of mitochondria to sustain energy output. Dynamic-related protein 1 (Drp1) and fission-related protein 1 (Fis1) are two GTPase proteins that are key regulators of mitochondrial fission. When mtROS levels increase, Fis 1 is predominantly activated and promotes the fragmentation of the mitochondrial network. ${ }^{23}$ The overexpression of Drp1 also facilitates excessive mitochondrial fission ${ }^{24}$ and results in cell apoptosis.

Under exposure to mild stress, mitochondria are elongated through the promotion of fusion, ${ }^{25}$ which can enhance ATP production and minimize stress-induced damage. Nevertheless, severe stress can severely injure mitochondria, and the impairment can compromise healthy mitochondria that share components with damaged mitochondria in the mitochondrial network. ${ }^{26}$ Increased expression of mitofusin (MFN) enhances mitochondrial elongation, ${ }^{12}$ whereas impaired mitochondria induce the generation of optic atrophy (OPA)-1 to degrade MFN-1 through the ubiquitin-proteasome system, resulting in mitochondrial fragmentation. In general, mitochondrial fragments tend to be eliminated. ${ }^{27}$ Studying CS exposure, researchers first found abnormal changes in mitochondrial morphology in primary airway epithelial cells derived from COPD patients, including crista defects, abnormal branches, and swollen and fragmented organelles. ${ }^{10,28}$ Using experimental data, researchers inferred that decreased oxygen consumption rates, decreased ATP levels, increased mtROS levels, reduced activity of the electron transport chain and loss of mitochondrial membrane potential are all contributors to abnormities in mitochondrial morphology in COPD patients. ${ }^{10,17,28-33}$ Innoxious doses of cigarette smoke extract (CSE) led to elevated metabolic activity accompanied by mitochondrial elongation in the absence of damaged mitochondria or ROS overgeneration in alveolar cells of mice. ${ }^{12}$ However, toxic doses of CSE caused fragmentation of mitochondria in human bronchial epithelial cells (HBECs) via the recruitment of Drp-1 to the damaged mitochondria. ${ }^{28,34}$ With the preservation of CS exposure at multiple time points, researchers found mitochondrial elongation 24 hours after exposure, whereas an identical dose of CSE induced fragmentation predominantly after 48 hours of exposure. ${ }^{35}$ Even for the same time (6 months) of CSE stimulation, bronchial epithelial cells exhibited different morphological alterations of mitochondria, including elongation and fragmentation. ${ }^{10}$ These results reveal that the alterations of fission and fusion in mitochondria are not inseparable or invariable but are coordinated to accommodate external stress. Researchers have observed morphological changes in mitochondria in the lung tissue from COPD patients, which reacted similarly to the different cell types under exposure to constant CSE, with both the mitochondrial elongation and fragmentation increased in the COPD samples. ${ }^{10,28}$ According to a previous study, the morphology of mitochondria does not precisely reflect the corresponding functions under severe or prolonged stress. The type and severity of the stimulus and the integrity of the mitochondrial quality control system, such as mitophagy, are also vital indicators of mitochondrial function, in addition to morphology. In general, the elongation of mitochondria reflects elevated mitochondrial function, while CS-induced elongation with decreased mitophagy reveals depressed mitochondrial functions and increased generation of ROS. ${ }^{12}$ Failure to alleviate these abnormities or attenuate the damage in a timely manner induces cellular senescence, ${ }^{28}$ and higher doses of CSE can induce cell death. ${ }^{29}$ In addition to the elimination of damaged mitochondria by mitophagy, mitochondrial biogenesis also regulates the quality of mitochondria via the AMP-activated protein kinase (AMPK)-peroxisome proliferator-activated receptor $\gamma$ coactivator-1-alpha (PGC1 $\alpha$ ) pathway, ${ }^{34}$ and oxidative stresses can stimulate this pathway. PGC1 $\alpha$ is upregulated in bronchial epithelial cells derived from COPD tissues, ${ }^{10}$ which may suggest increased mitochondrial biogenesis in COPD, which would indicate an adaptive response to oxidative stresses. However, under perennial CS stimulation, the upregulation of mitochondrial biogenesis with increased expression of PGCl $\alpha$ causes cellular senescence. ${ }^{36}$ 
Using COPD epithelial cells, researchers demonstrated elevated numbers of mitochondria with cristae depletion, which indicates damaged mitochondria in COPD. Compared its effects of other diseases, the PTEN-induced putative kinase 1 (PINK1)/parkin RBR E3 ubiquitin protein ligase (PRKN) pathway plays a pivotal role in mitophagy in COPD. PEKN expression is decreased in COPD lung tissue, and PRKN knockout enhances airway wall thickening due to changes causing emphysema after CS exposure in COPD mice, compared with its effect in wild type (WT) mice. ${ }^{3,35}$ In contrast to these reports, Mizumura proved that CS exposure activates mitophagy, leading to cell death in a mouse model. ${ }^{17}$ Mizumura demonstrated that the upregulation of PINK1 and PINK1-/- mice were protected against emphysematous changes during CS stimulus. Mizumura also found elevated expression of the necroptosis regulator receptor-interacting serine-threonine kinase 3 (RIP3) and inferred that CS might increase the level of mitophagy and induce necrotic cell death, changes dependent on upregulated mitophagy in the pathogenesis of COPD. Several studies have indicated that mtROS are not involved in the mitochondrial translocation of PRKN, but Bin Xiao indicated that the stimulation of carbonyl cyanide m-chlorophenylhydrazone (CCCP) is essential for mitophagy. ${ }^{37}$ Treatment with CCCP can induce both mitochondrial depolarization and the accumulation of ROS. An objective analysis suggests that different experimental conditions may have led to the discrepancies between these two results, and the concentration and stimulation time might have also influenced the results.

\section{Mitochondrial Dysfunction in Inflammatory Response Which Contributes to COPD Pathogenesis} Mitochondria play vital roles in proinflammatory signaling, and proinflammatory factors similarly influence mitochondrial functions. ${ }^{38}$ Previous studies have shown that high levels of mtROS, extracellular ATP, and mtDNA release inflict inflammatory responses that lead to mitochondrial dysfunction. Excessive oxidative stresses lead to the recruitment of inflammatory cells to the lung, including neutrophils, CD8+ T lymphocytes and alveolar macrophages, and these cells affect the structures and functions of pulmonary epithelial cells. ${ }^{39}$ The imbalance of the protease/antiprotease system in the process of inflammation facilitates the release of proteolytic enzymes from neutrophils and macrophages. ${ }^{40}$ Under severe stresses, mitochondria generate a large number of mtROS that cause an inflammatory response via several mechanisms, including tumor necrosis factor receptors (TNFRs), mitogen-activated protein kinase (MAPK), RIG-I-like receptors and NLR family pyrin domain-containing 3 (NLRP3) inflammasome signals. ${ }^{5,41-43}$ Bulua demonstrated that the inhibition of mtROS generation reduced the levels of proinflammatory mediators, such as interleukin (IL)-6 and tumor necrosis factor (TNF), in cells isolated from patients with TNFR1-associated periodic syndrome. ${ }^{5}$ The released ATP activates purine receptors ( $\mathrm{P} 2 \mathrm{X}$ and $\mathrm{P} 2 \mathrm{Y})$ in an autocrine and paracrine manner, and the activation of these purine receptors leads to the generation of ROS, proinflammatory mediators and chemokines. ${ }^{44,45}$ Additionally, mtDNA released in the cytosol and circulation can act as mitochondrial damage-associated molecular patterns (DAMPs), which induce inflammasome activation. ${ }^{46}$ Further study shows that mtDNA-related inflammasome activation is related to the cellautonomous activation of specific pattern recognition receptors such as Toll-like receptor 9 (TLR9). ${ }^{47}$ Zhou found that the deletion of NLRP3 suppresses the increase in interleukin (IL)- $1 \beta$ caused by mtROS accumulation. ${ }^{43}$

Mitophagy serves as a predominant part of the mitochondrial quality control system, which also plays a vital role in the inflammatory response caused by mitochondrial dysfunction. Zhong demonstrated that macrophages derived from PRKN-/- mice secrete IL-1 $\beta$ and stimulate NLRP3 activators. ${ }^{48}$ Then, they revealed that the mitochondrial damage induced by NLRP3 activators may contribute to PRKN-dependent mitophagy. However, Allam found that macrophages isolated from PRKN-/- mice do not produce lower levels of IL-1 $\beta$ in response to NLRP3stimulating treatments, but the underlying mechanism of mitophagy in regulating inflammasome activation is still unclear. ${ }^{49}$ Researchers have found that $N$-glycanase 1 (NGLY1)-deficient human and mouse cells exhibit activated nucleic acid-sensing pathways that induce an elevated expression of IFN-stimulated genes (ISGs). ${ }^{50}$ This study elucidated the protective function of NGLY1 by promoting the expression of nuclear factor erythroid 2 like 1 (NRF1), a transcriptional factor, and restoring mitophagy and mitochondrial homeostasis to relieve the inflammatory response. Notably, inflammation is a critical driver of Parkinson's disease (PD) pathology. ${ }^{51}$ Upon the knockout of PINK1 or PRKN, exhaustive exercise and mutational stress of mtDNA activated the DNAsensing cGAS-STING pathway and led to an inflammatory 
phenotype. ${ }^{52}$ Strikingly, in this study, after exhaustive exercise, the levels of several cytokines were elevated, including IL-6, IL-12, IL-13, interferon- $\beta$ (IFN $\beta$ ), $\mathrm{C}-\mathrm{X}-\mathrm{C}$ motif chemokine ligand 1 (CXCL1), C-C motif chemokine ligand 2 (CCL2) and CCL4, in the serum of PINK1-/- or PRKN-/- mice. Mannam P demonstrated that mitogen-activated protein kinase kinase 3 (MKK3)knockout mice are resistant to CS-induced inflammation and mitochondrial dysfunction. ${ }^{53}$ Prior to this study, Srivastava A indicated that MKK3 is a negative regulator of mitochondrial health. ${ }^{54}$ The MKK3-knockout mice were protected against inflammatory injuries of the lung $^{55,56}$ and showed increased mitophagy compared to WT mice, enhancing the number of healthy mitochondria. ${ }^{54}$ After CS exposure, the WT mice in this study showed more significant upregulation of serum INF $\gamma$, IL-6, calcitonin-related polypeptide alpha (KC), nuclear factor kappa $\mathrm{B}(\mathrm{NF} \kappa \mathrm{B})$ and $\mathrm{TNF} \alpha$ than the MKK3-/- mice. Researchers collected lung tissue specimens from COPD patients and found higher p-MMK3 (the active form) levels than in non-COPD patients. These results revealed that $\mathrm{MKK} 3$ can be regarded as a therapeutic target to alleviate inflammation and mitochondrial damage in COPD.

\section{The Role of Mitochondrial Homeostasis in CS-Induced Cellular Senescence}

Mitochondria regulate cell functions by modulating not only the generation of energy but also aging-associated phenotypes, such as cellular senescence. ${ }^{57}$ Cellular components, including proteins, DNA and lipids, are all affected by the accumulation of damaged mitochondria with excessive production of ROS. ${ }^{57}$ Overwhelming mtROS levels lead to telomere shortening by generating single-strand breaks in telomeric regions, which may suggest a mechanism by which ROS-mediated cellular senescence regulates telomere health. ${ }^{58}$ Old age is an acknowledged risk factor that significantly influences the process of COPD. ${ }^{59}$ It has been established that mitochondrial functions decline with aging in model organisms, ${ }^{60}$ but this phenomenon is also observed in aging people and may lead to several age-related diseases. ${ }^{61}$ Researchers have demonstrated that mtDNA mutations contribute to mammalian aging by mutating the amino acid at position 257 (D257A) in the sole mtDNA polymerase. ${ }^{20,62}$ Peroxisome proliferator-activated receptor $\gamma$ coactivator-
$1-\alpha(\mathrm{PGC}-1 \alpha)$ is the main coactivator of mitochondrial biogenesis, ${ }^{63}$ modulating the activity of nuclear respiratory factors NRF1 and NRF2 and mitochondrial transcription factor $A$ (TFAM) involved in the regulation of mitochondrial biogenesis. ${ }^{64}$ Elevated levels of PGC-1 $\alpha$ in mouse skeletal muscle suppressed the development of agedependent sarcopenia. ${ }^{65}$ Kaplon revealed the relationship between mitochondrial metabolism and cellular senescence induced by oncogenes and the related increase in the oxidation of pyruvate, resulting in increased mtROS of cells in a senescent state. ${ }^{66}$ The phosphorylation of pyruvate enhances the activity of the mitochondrial pyruvate dehydrogenase (PDH) complex, and the PDH complex is also regulated by mitochondrial sirtuins, especially sirtuin (SIRT)-3 and SIRT4. ${ }^{67,68}$

Parkinson's disease is known as an aging-associated disease, and loss-of-function mutations in either PINK1 or PRKN can result in the early onset of Parkinson's disease. ${ }^{69}$ The deletion of PRKN in Drosophila decreased the fly lifespan, ${ }^{70}$ and the overexpression of PRKN expanded the fly lifespan without restricting fly fertility or food consumption. ${ }^{71}$ LON protease, known as a vital protein for mitochondrial protein homeostasis, is reported to regulate the mitochondrial level of PINK $1,{ }^{72}$ and the decreased expression of LON protease is reported to be related to reduced mitochondrial function and exacerbated aging. ${ }^{73}$ Although the link between aging and cellular senescence is not simply linear, we obtained some enlightening results. Many studies have shown that cellular senescence contributes to the complex pathogenesis of COPD. ${ }^{74}$ In the airway epithelial cells of mice, the overexpression of SIRT1, which can activate mitochondrial biogenesis, shows resistance to the CS-induced cellular senescence induced by deacetylating forkhead box $\mathrm{O3}$ (FOXO3). ${ }^{75}$ FOXO3 had been previously reported to enhance mitochondrial function and alleviate CS-induced senescence. ${ }^{76}$ It is noteworthy that the PINK1/PRKN pathway still plays a crucial role in CS-induced senescence in airway epithelial cells. ${ }^{3,4,12}$ Ito $\mathrm{S}$ elucidated the regulatory mechanism among ROS, mitophagy and cellular senescence under CS exposure, showing that reduced levels of PRKN but increased levels of PINK1 were significantly observed in COPD lungs compared with these levels in non-COPD lungs. ${ }^{3}$ Ahmad T performed further research to demonstrate a step-by-step regulatory pattern in which CSE induces elevated production of ROS, and the accumulated ROS attenuate mitophagy, which eventually leads to cellular senescence. ${ }^{12}$ Ahmad $\mathrm{T}$ showed that increased 
ROS and DNA damage foci formation were driven by CSE-mediated perinuclear mitochondrial accumulation and that CSE impaired the effect of CCCP, a known PRKN mitochondrial translocation agent, which indicated that CSE led to diminished mitophagy. However, a mitochondria-targeted antioxidant ameliorated cellular senescence, indicating that mtROS establish an indispensable connection with mitophagy impairment and cellular senescence in lung airway epithelial cells. Interestingly, Araya et al designed a prkn-knockout (KO) to verify that PRKN protein levels are the rate-limiting factors in PNIK1-PRKN-mediated mitophagy under CS exposure, and the findings indicated that PRKN might become a therapeutic target in the process of COPD. ${ }^{4}$

\section{Mitophagy-Dependent Necroptosis Contribute to Emphysema of COPD} Emphysema is a common manifestation of COPD, ${ }^{59}$ which is considered to be caused by smoking-induced epithelial cell death. ${ }^{77}$ Apoptosis is proposed to be an underlying mechanism leading to emphysema in COPD, whereas apoptosis is usually not accompanied by inflammation and is associated with no or few DAMPs. Nevertheless, necroptosis is normally regarded as a predominant reason for emphysema with COPD because a massive number of DAMPs is released from damaged cells. ${ }^{78}$ Emerging studies demonstrate that there is a genetically programmed and regulated form of necrosis, termed necroptosis, that is dependent on receptorinteracting protein kinase 3 (RIPK3) and differs from apoptosis and necrosis. ${ }^{79-81}$ Autophagy, including selective autophagy, is usually considered a protective mechanism, and autophagic cell death is not deemed a type of programmed cell death. ${ }^{82,83}$ However, autophagy indeed modulates other cell death pathways. ${ }^{84,85}$ Mizumura $\mathrm{K}$ showed that the mitophagy inhibitor Mdivi-1 and the deletion of PINK1 protect against mitochondrial dysfunction and cell death and downregulate the phosphorylation of mixed lineage kinase domains such as pseudokinase (MLKL), which is a substrate for RIP3 (a key regulator of necroptosis). ${ }^{17}$ Moreover, PINK1-/- mice were resistant to CS-induced airspace enlargement, mucociliary clearance (MCC) disruption and mitochondrial injuries in this study. Importantly, the overexpression of PINK1 and RIP3 was observed in epithelial cells isolated from COPD patients. These manifestations indicate that mitophagydependent necroptosis might be a therapeutic target for
COPD. In addition, Mizumura $\mathrm{K}$ reported that in lung epithelial cells exposed to CS, sphingolipids regulated mitophagy and necroptosis and that the inhibition of ceramide-generating acid sphingomyelinase decreased both necroptosis and the CS-induced phosphorylation of PINK1. ${ }^{86}$

\section{Mitophagy and Innate Immunity in Infection}

Exacerbations of COPD has been proven to be related to susceptibility to infections. ${ }^{59}$ Researchers constructed a large observational study to show that the colonization of Pseudomonas aeruginosa increased the risk of hospitalization for exacerbated COPD, increasing COPD mortality. ${ }^{87}$ Deficient mitophagy is associated with immune cell homeostasis and inflammatory cytokine secretion, which contribute to autoimmune disease pathologies. ${ }^{14}$ Pathogens can directly modulate mitophagy, and pathogenic components can influence the innate immune response, which promotes pathogen replication in host cells. ${ }^{15,16}$ In a model of polymicrobial sepsis, the PINK1/PRKN pathway plays a crucial role in protecting the host against inflammation, and host susceptibility and inflammasome formation are upregulated when PINK1 or PRKN is deficient. ${ }^{88}$ Similarly, PRKN protects against sepsis-induced cardiac contractility by activating mitophagy. ${ }^{89}$ Interestingly, several pathogens promote mitophagy during infection by inducing mitochondrial fission. ${ }^{90,91}$ Zhou demonstrated that viral infection inhibits PINK1 to modulate the antiviral response by mediating RIG-I-like receptor (RLR) signaling, which affects with TNF receptor-associated factor (TRAF) 3 by suppressing its degradation. ${ }^{92}$ Using a constructed an intestinal bacterial infection model, researchers found that PINK1-/- mice exhibit autoimmunity and mitochondria-specific CD8+ $\mathrm{T}$ cell responses. ${ }^{93}$ Various kinases are involved in the functions of innate immunity and mitophagy, such as TANK-binding kinase 1 (TBK1). TBK1 can activate the synthesis of IFN regulatory factor (IRF)-3 and type I IFN, ${ }^{94}$ and the autophosphorylation of TBK1 is an important step for mitophagy upon the ubiquitination of mitochondrial fragments. ${ }^{95}$ In addition, the phosphorylation of optineurin (OPTN) by TBK1 promotes the binding efficiency of OPTN to ubiquitin (Ub) chains, which enhances mitophagy. ${ }^{96}$ These findings implicate TBK1 as a key mediator of innate immunity and the activation of mitophagy. Notably, Jnk2-deficient mice express 
inefficient mitophagy and elevated susceptibility to sepsis. ${ }^{97}$ The deletion of MKK3 also protects against sepsis by enabling mitochondrial biogenesis and mitophagy recovery, revealing that MKK3 plays a crucial role in the mitochondrial quality control system. ${ }^{55}$

Recent studies have shown the functions of calcium binding and coiled-coil domain 2 (CALCOCO2/NDP52), known as a key factor in the early step of PINK1/PRKNdependent mitophagy, ${ }^{98}$ and $\mathrm{p} 62$ in regulating infection by coxsackievirus B3 and mycobacteria. ${ }^{99,100}$ NDP52 plays a pivotal role by degrading autophagy-mediated mitochondrial antiviral-signaling protein (MAVS), resulting in the activation of NF- $\mathrm{BB}$ and IRF3, inhibiting type I IFN signaling. ${ }^{99}$ Mitochondrial fragmentation and the generation of mitophagy-related autophagosomes are measures by which coxsackievirus B3 escapes the host immune response. $^{101,102}$ When infected with an RNA virus, leucine-rich repeat-containing protein 25 (LRRC25) ${ }^{104}$ activates p62 to induce selective autophagy-mediated RIG-I degradation, while LRRC59 ${ }^{105}$ inhibits p62 in the same way. Furthermore, OPTN suppresses the intestinal inflammatory response in Salmonella infection by elevating the capacity of clearance ${ }^{105}$ and increases cell sensitivity to Citrobacter colitis and Escherichia colitis. ${ }^{106}$ In addition, NOD-like receptor X1 (NLRX1) is a newly discovered mitophagy receptor containing an LIR motif that associates with LC3, which is the target of Listeria monocytogenes for inducing mitophagy and evading the host killing effect. $^{107}$ Overall, pathogens target critical mitophagy receptors or innate immune signaling factors under stress conditions to regulate the functions of the innate immune system.

\section{Conclusion}

COPD is a predominant cause of chronic morbidity and mortality throughout the world and is thus considered a significant public health issue that is both preventable and treatable. Cigarette smoking is the most commonly encountered risk factor for COPD. CS-induced oxidative stress acts on mitochondria, leading to alterations in their morphology, function and quality control, which accelerate the generation and accumulation of mtROS. Loss of cristae, formation of abnormal branches, swollen and fragmented organelles are generally found in primary airway epithelial cells isolated from COPD patients. These changes in morphology result in damaged mitochondrial functions, such as decreased production of ATP, increased levels of ROS and reduced activity of the electron transport chain. Mitophagy, as a selective autophagy to eliminate impaired mitochondria, is also observed to be impaired in CS-stimulated experimental models and COPD patients. PRKN is the pivotal regulatory factor of mitophagy that is downregulated in COPD lung tissue, and the knockout of PRKN enhances the resistance of lung airway epithelial cells to CS-induced damage. Inflammation and necroptosis are the fundamental pathological changes caused by COPD, and mitophagy plays an important protective role in these variations. Age, regarded as one of the risk factors for COPD, is usually associated with an elevated level of cellular senescence in COPD patients. Excessive mtROS are the primary pathogenic factors in CS-induced cellular senescence, which accumulate because of impaired mitophagy during CS exposure. Researchers have indicated that PRKN may be a mechanistic target in the cellular senescence of COPD because it regulates mitophagy, as shown by prknknockout mice being protected against CS-induced cell senescence. Infections also play a predominant role in the exacerbation of COPD, and defective mitophagy, as an underlying outcome of various pathogen infections, may influence the innate immune response to facilitate pathogen replication and colonization. Overall, mitophagy exerts several effects on pathological alterations, and various related genes or molecules can be expected to be used as therapeutic targets in the future.

\section{Abbreviations}

AC, acetyl; CCCP, carbonyl cyanide $m$-chlorophenylhydrazone; CCL2, C-C motif chemokine ligand 2; COPD, Chronic obstructive pulmonary disease; $\mathrm{CS}$, cigarette smoke; CSE, cigarette smoke extract; CXCL1, C-X-C motif chemokine ligand 1; DAMP, damage-associated molecular patterns; Drp1, dynamic-related protein 1; Fis1, fission-related protein 1; FOXO3, forkhead box O3; HBEC, human bronchial epithelial cell; IFN, interferon; IL, interleukin; IRF3, IFN regulatory factor 3; ISG, IFN-stimulated gene; $\mathrm{KC}$, calcitonin related polypeptide alpha; KO, knockout; LRRC25, leucine-rich repeat containing protein 25; MAPK, mitogen activated protein kinase; MAVS, mitochondrial antiviral-signaling protein; MCC, mucociliary clearance; MFN, mitofusin; MKK3, mitogen activated protein kinase kinase 3; MLKL, mixed lineage kinase domain like pseudokinase; mtDNA, mitochondrial DNA; mtROS, mitochondrial ROS; NDP52, calcium binding and coiled-coil domain 2; NFאB, nuclear factor kappa B; NGLY1, N-glycanase 1; NLRP3, NLR family pyrin domain containing 3; NLRX1, NOD-like receptor X1; NRF1, nuclear 
factor, erythroid 2 like 1; OPA, optic atrophy; OPTN, optineurin; PD, Parkinson's disease; PDH, pyruvate dehydrogenase; PGC-1 $\alpha$, peroxisome proliferator-activated receptor $\gamma$ coactivator-1 $\alpha$; PINK1, PTEN-induced putative kinase 1; PRKN, parkin RBR E3 ubiquitin protein ligase; RIP3, receptor-interacting serine-threonine kinase 3; RIPK3, receptorinteracting protein kinase 3; RLR, RIG-I like receptor; ROS, reactive oxygen species; SIRT, sirtuin; TBK1, TANK binding kinase 1; TFAM, mitochondrial transcription factor A; TLR9, toll like receptor 9; TNFR, tumor necrosis factor receptor; TRAF, TNF receptor-associated factor; WT, wild type.

\section{Author Contributions}

All authors made a significant contribution to the work reported, whether that is in the conception, study design, execution, acquisition of data, analysis and interpretation, or in all these areas; took part in drafting, revising or critically reviewing the article; gave final approval of the version to be published; have agreed on the journal to which the article has been submitted; and agree to be accountable for all aspects of the work.

\section{Funding}

This study was supported by grants from the Science and Technology Foundation of Shandong Province (grant numbers 2015GGH318002, 2007GG3002008, and 2015GSF118064).

\section{Disclosure}

The authors declare that they have no conflicts of interest for this work.

\section{References}

1. Dal-Ré R. Worldwide behavioral research on major global causes of mortality. Health Educ Behav. 2011;38(5):433 440.

2. Araya J, Cambier S, Markovics JA, et al. Squamous metaplasia amplifies pathologic epithelial-mesenchymal interactions in COPD patients. J Clin Invest. 2007;117(11):3551 3562.

3. Ito S, Araya J, Kurita Y, et al. PARK2-mediated mitophagy is involved in regulation of HBEC senescence in COPD pathogenesis. Autophagy. 2015;11(3):547-559.

4. Araya J, Tsubouchi K, Sato N, et al. PRKN-regulated mitophagy and cellular senescence during COPD pathogenesis. Autophagy 2019;15 (3):510-526.

5. Bulua AC, Simon A, Maddipati R, et al. Mitochondrial reactive oxygen species promote production of proinflammatory cytokines and are elevated in TNFR1-associated periodic syndrome (TRAPS). $J$ Exp Med. 2011;208(3):519-533.

6. Minutoli L, Puzzolo D, Rinaldi M, et al. ROS-mediated NLRP3 inflammasome activation in brain, heart, kidney, and testis ischemia/ reperfusion injury. Oxid Med Cell Longev. 2016;2016:2183026.

7. Park J, Choi H, Min JS, et al. Mitochondrial dynamics modulate the expression of pro-inflammatory mediators in microglial cells. J Neurochem. 2013;127(2):221 232.
8. Yue L, Yao H. Mitochondrial dysfunction in inflammatory responses and cellular senescence: pathogenesis and pharmacological targets for chronic lung diseases. Br J Pharmacol. 2016;173 (15):2305-2318.

9. Youle RJ, Narendra DP. Mechanisms of mitophagy. Nat Rev Mol Cell Biol. 2011;12(1):9-14.

10. Hoffmann RF, Zarrintan S, Brandenburg SM, et al. Prolonged cigarette smoke exposure alters mitochondrial structure and function in airway epithelial cells. Respir Res. 2013;14(1):97.

11. Ballweg K, Mutze K, Konigshoff M, et al. Cigarette smoke extract affects mitochondrial function in alveolar epithelial cells. Am J Physiol Lung Cell Mol Physiol. 2014;307(11):L895-L907.

12. Ahmad T, Sundar IK, Lerner CA, et al. Impaired mitophagy leads to cigarette smoke stress-induced cellular senescence: implications for chronic obstructive pulmonary disease. FASEB $J$. 2015;29(7):2912-2929.

13. Aghapour M, Remels AHV, Pouwels SD, et al. Mitochondria: at the crossroads of regulating lung epithelial cell function in chronic obstructive pulmonary disease. Am J Physiol Lung Cell Mol Physiol. 2020;318(1):L149-L164.

14. Montava-Garriga L, Ganley IG. Outstanding questions in mitophagy: what we do and do not know. J Mol Biol. 2020;432 (1):206-230.

15. Jin HS, Suh HW, Kim SJ, et al. Mitochondrial control of innate immunity and inflammation. Immune Netw. 2017;17(2):77-88.

16. Mohanty A, Tiwari-Pandey R, Pandey NR. Mitochondria: the indispensable players in innate immunity and guardians of the inflammatory response. J Cell Commun Signal. 2019;13(3):303-318.

17. Mizumura K, Cloonan SM, Nakahira K, et al. Mitophagydependent necroptosis contributes to the pathogenesis of COPD. J Clin Invest. 2014;124(9):3987-4003.

18. Antico Arciuch VG, Elguero ME, Poderoso JJ, et al. Mitochondrial regulation of cell cycle and proliferation. Antioxid Redox Signal. 2012;16(10):1150-1180.

19. Yakes FM, Van Houten B. Mitochondrial DNA damage is more extensive and persists longer than nuclear DNA damage in human cells following oxidative stress. Proc Natl Acad Sci USA. 1997;94(2):514-519.

20. Kujoth GC, Hiona A, Pugh TD, et al. Mitochondrial DNA mutations, oxidative stress, and apoptosis in mammalian aging. Science. 2005;309(5733):481-484.

21. Bolisetty S, Jaimes EA. Mitochondria and reactive oxygen species: physiology and pathophysiology. Int J Mol Sci. 2013;14 (3):6306-6344.

22. Trian T, Benard G, Begueret $\mathrm{H}$, et al. Bronchial smooth muscle remodeling involves calcium-dependent enhanced mitochondrial biogenesis in asthma. J Exp Med. 2007;204(13):3173-3181.

23. Reddy PH. Mitochondrial medicine for aging and neurodegenerative diseases. Neuromolecular Med. 2008;10(4):291-315.

24. Frank S, Gaume B, Bergmann-Leitner ES, et al. The role of dynamin-related protein 1 , a mediator of mitochondrial fission, in apoptosis. Dev Cell. 2001;1(4):515-525.

25. Zemirli N, Morel E, Molino D. Mitochondrial dynamics in basal and stressful conditions. Int J Mol Sci. 2018;19(2):E564.

26. Youle RJ, van der Bliek AM. Mitochondrial fission, fusion, and stress. Science. 2012;337(6098):1062-1065.

27. Ehses S, Raschke I, Mancuso G, et al. Regulation of OPA1 processing and mitochondrial fusion by m-AAA protease isoenzymes and OMA1. J Cell Biol. 2009;187(7):1023-1036.

28. Hara $\mathrm{H}$, Araya $\mathrm{J}$, Ito $\mathrm{S}$, et al. Mitochondrial fragmentation in cigarette smoke-induced bronchial epithelial cell senescence. Am J Physiol Lung Cell Mol Physiol. 2013;305(10):L737-L746.

29. van der Toorn M, Rezayat D, Kauffman HF, et al. Lipid-soluble components in cigarette smoke induce mitochondrial production of reactive oxygen species in lung epithelial cells. Am J Physiol Lung Cell Mol Physiol. 2009;297(1):L109-L114. 
30. Agarwal AR, Yin F, Cadenas E. Short-term cigarette smoke exposure leads to metabolic alterations in lung alveolar cells. Am J Respir Cell Mol Biol. 2014;51(2):284-293.

31. Cloonan SM, Choi AM. Mitochondria in lung disease. J Clin Invest. 2016;126(3):809-820.

32. Kosmider B, Lin CR, Karim L, et al. Mitochondrial dysfunction in human primary alveolar type II cells in emphysema. EBioMedicine. 2019;46:305-316.

33. Malinska D, Szymański J, Patalas-Krawczyk P, et al. Assessment of mitochondrial function following short- and long-term exposure of human bronchial epithelial cells to total particulate matter from a candidate modified-risk tobacco product and reference cigarettes. Food Chem Toxicol. 2018;115:1-12.

34. Zhang Z, Cheng X, Yue L, et al. Molecular pathogenesis in chronic obstructive pulmonary disease and therapeutic potential by targeting AMP-activated protein kinase. J Cell Physiol. 2018;233(3):1999-2006.

35. Hara H, Kuwano K, Araya J. Mitochondrial quality control in COPD and IPF. Cells. 2018;7(8):E86.

36. Summer R, Shaghaghi H, Schriner D, et al. Activation of the mTORC1/PGC-1 axis promotes mitochondrial biogenesis and induces cellular senescence in the lung epithelium. Am J Physiol Lung Cell Mol Physiol. 2019;316(6):L1049-L1060.

37. Xiao B, Deng X, Lim GGY, et al. Superoxide drives progression of Parkin/PINK1-dependent mitophagy following translocation of parkin to mitochondria. Cell Death Dis. 2017;8(10):e3097.

38. López-Armada MJ, Riveiro-Naveira RR, Vaamonde-García C, et al. Mitochondrial dysfunction and the inflammatory response. Mitochondrion. 2013;13(2):106-118.

39. Goldkorn T, Filosto S. Lung injury and cancer: mechanistic insights into ceramide and EGFR signaling under cigarette smoke. Am J Respir Cell Mol Biol. 2010;43(3):259-268.

40. Jiang Y, Wang $\mathrm{X}, \mathrm{Hu} \mathrm{D}$. Mitochondrial alterations during oxidative stress in chronic obstructive pulmonary disease. Int J Chron Obstruct Pulmon Dis. 2017;13(12):1153-1162.

41. Emre Y, Hurtaud C, Nübel T, et al. Mitochondria contribute to LPS-induced MAPK activation via uncoupling protein UCP2 in macrophages. Biochem J. 2007;402(2):271-278.

42. Tal MC, Sasai M, Lee HK, et al. Absence of autophagy results in reactive oxygen species-dependent amplification of RLR signaling. Proc Natl Acad Sci U S A. 2009;106(8):2770-2775.

43. Zhou R, Yazdi AS, Menu P, et al. A role for mitochondria in NLRP3 inflammasome activation. Nature. 2011;469 (7329):221-225.

44. Bours MJ, Dagnelie PC, Giuliani AL, et al. P2 receptors and extracellular ATP: a novel homeostatic pathway in inflammation. Front Biosci. 2011;3:1443-1456.

45. Cauwels A, Rogge E, Vandendriessche B, et al. Extracellular ATP drives systemic inflammation, tissue damage and mortality. Cell Death Dis. 2014;5:e1102.

46. Kepp O, Galluzzi L, Kroemer G. Mitochondrial control of the NLRP3 inflammasome. Nat Immunol. 2011;12(3):199-200.

47. Oka T, Hikoso S, Yamaguchi O, et al. Mitochondrial DNA that escapes from autophagy causes inflammation and heart failure. Nature. 2012;485(7397):251-255.

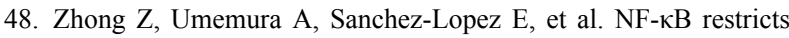
inflammasome activation via elimination of damaged mitochondria. Cell. 2016;164(5):896-910.

49. Allam R, Lawlor KE, Yu EC, et al. Mitochondrial apoptosis is dispensable for NLRP3 inflammasome activation but non-apoptotic caspase- 8 is required for inflammasome priming. EMBO Rep. 2014;15(9):982-990.

50. Yang K, Huang R, Fujihira H, et al. N-glycanase NGLY1 regulates mitochondrial homeostasis and inflammation through NRF1. J Exp Med. 2018;215(10):2600-2616.
51. Herrero MT, Estrada C, Maatouk L, et al. Inflammation in parkinson's disease: role of glucocorticoids. Front Neuroanat. 2015;9:32.

52. Sliter DA, Martinez J, Hao L, et al. Parkin and PINK1 mitigate STI NG-induced inflammation. Nature. 2018;561(7722):258-262.

53. Mannam P, Rauniyar N, Lam TT, et al. MKK3 influences mitophagy and is involved in cigarette smoke-induced inflammation. Free Radic Biol Med. 2016;101:102-115.

54. Srivastava A, McGinniss J, Wong Y, et al. MKK3 deletion improves mitochondrial quality. Free Radic Biol Med. 2015;87:373-384.

55. Mannam P, Shinn AS, Srivastava A, et al. MKK3 regulates mitochondrial biogenesis and mitophagy in sepsis-induced lung injury. Am J Physiol Lung Cell Mol Physiol. 2014;306(7):L604L619.

56. Mannam P, Zhang X, Shan P, et al. Endothelial MKK3 is a critical mediator of lethal murine endotoxemia and acute lung injury. J Immunol. 2013;190(3):1264-1275.

57. Bratic A, Larsson NG. The role of mitochondria in aging. J Clin Invest. 2013;123(3):951-957.

58. Birch J, Barnes PJ, Passos JF. Mitochondria, telomeres and cell senescence: implications for lung ageing and disease. Pharmacol Ther. 2018;183:34-49.

59. Singh D, Agusti A, Anzueto A, et al. Global strategy for the diagnosis, management, and prevention of chronic obstructive lung disease: the GOLD science committee report 2019. Eur Respir J. 2019;53(5):1900164.

60. Rockstein M, Brandt KF. Enzyme changes in flight muscle correlated with aging and flight ability in the male housefly. Science. 1963;139(3559):1049-1051.

61. Petersen KF, Befroy D, Dufour S, et al. Mitochondrial dysfunction in the elderly: possible role in insulin resistance. Science. 2003;300(5622):1140-1142.

62. Trifunovic A, Wredenberg A, Falkenberg M, et al. Premature ageing in mice expressing defective mitochondrial DNA polymerase. Nature. 2004;429(6990):417-423.

63. Ruas JL, White JP, Rao RR, et al. A PGC-1 $\alpha$ isoform induced by resistance training regulates skeletal muscle hypertrophy. Cell. 2012;151(6):1319-1331.

64. Austin S, St-Pierre J. PGC1 $\alpha$ and mitochondrial metabolismemerging concepts and relevance in ageing and neurodegenerative disorders. J Cell Sci. 2012;125(Pt 21):4963-4971.

65. Wenz T, Rossi SG, Rotundo RL, et al. Increased muscle PGC-1alpha expression protects from sarcopenia and metabolic disease during aging. Proc Natl Acad Sci U S A. 2009;106 (48):20405-20410.

66. Kaplon J, Zheng L, Meissl K, et al. A key role for mitochondrial gatekeeper pyruvate dehydrogenase in oncogene-induced senescence. Nature. 2013;498(7452):109-112.

67. Mathias RA, Greco TM, Oberstein A, et al. Sirtuin 4 is a lipoamidase regulating pyruvate dehydrogenase complex activity. Cell. 2014;159(7):1615-1625.

68. Fan J, Shan C, Kang HB, et al. Tyr phosphorylation of PDP1 toggles recruitment between ACAT1 and SIRT3 to regulate the pyruvate dehydrogenase complex. Mol Cell. 2014;53(4):534-548.

69. Durcan TM, Fon EA. The three 'P's of mitophagy: PARKIN, PINK1, and post-translational modifications. Genes Dev. 2015;29 (10):989-999.

70. Greene JC, Whitworth AJ, Kuo I, et al. Mitochondrial pathology and apoptotic muscle degeneration in Drosophila parkin mutants. Proc Natl Acad Sci U S A. 2003;100(7):4078-4083.

71. Rana A, Rera M, Walker DW. Parkin overexpression during aging reduces proteotoxicity, alters mitochondrial dynamics, and extends lifespan. Proc Natl Acad Sci U S A. 2013;110 (21):8638-8643. 
72. Thomas RE, Andrews LA, Burman JL, et al. PINK1-parkin pathway activity is regulated by degradation of PINK1 in the mitochondrial matrix. PLoS Genet. 2014;10(5):e1004279.

73. Quirós PM, Langer T, López-Otín C. New roles for mitochondrial proteases in health, ageing and disease. Nat Rev Mol Cell Biol. 2015;16(6):345-359.

74. Barnes PJ, Baker J, Donnelly LE. Cellular senescence as a mechanism and target in chronic lung diseases. Am J Respir Crit Care Med. 2019;200(5):556-564.

75. Yao H, Chung S, Hwang JW, et al. SIRT1 protects against emphysema via FOXO3-mediated reduction of premature senescence in mice. J Clin Invest. 2012;122(6):2032-2045.

76. Hagenbuchner J, Ausserlechner MJ. Mitochondria and FOXO3: breath or die. Front Physiol. 2013;4:147.

77. Mizumura K, Maruoka S, Shimizu T, et al. Autophagy, selective autophagy, and necroptosis in COPD. Int J Chron Obstruct Pulmon Dis. 2018;13:3165-3172.

78. Pasparakis M, Vandenabeele P. Necroptosis and its role in inflammation. Nature. 2015;517(7534):311-320.

79. Cho YS, Challa S, Moquin D, et al. Phosphorylation-driven assembly of the RIP1-RIP3 complex regulates programmed necrosis and virus- induced inflammation. Cell. 2009;137 (6):1112-1123.

80. He S, Wang L, Miao L, et al. Receptor interacting protein kinase-3 determines cellular necrotic response to TNF-alpha. Cell. 2009;137(6):1100-1111.

81. Zhang DW, Shao J, Lin J, et al. RIP3, an energy metabolism regulator that switches TNF-induced cell death from apoptosis to necrosis. Science. 2009;325(5938):332-336.

82. Schweichel JU, Merker HJ. The morphology of various types of cell death in prenatal tissues. Teratology. 1973;7(3):253-266.

83. Kroemer G, Levine B. Autophagic cell death: the story of a misnomer. Nat Rev Mol Cell Biol. 2008;9(12):1004-1010.

84. Bonapace L, Bornhauser BC, Schmitz M, et al. Induction of autophagy-dependent necroptosis is required for childhood acute lymphoblastic leukemia cells to overcome glucocorticoid resistance. J Clin Invest. 2010;120(4):1310-1323.

85. Khan MJ, Rizwan Alam M, Waldeck-Weiermair M, et al. Inhibition of autophagy rescues palmitic acid-induced necroptosis of endothelial cells. J Biol Chem. 2012;287(25):21110-21120.

86. Mizumura K, Justice MJ, Schweitzer KS, et al. Sphingolipid regulation of lung epithelial cell mitophagy and necroptosis during cigarette smoke exposure. FASEB J. 2018;32(4):1880-1890.

87. Eklöf J, Sørensen R, Ingebrigtsen TS, et al. Pseudomonas aeruginosa and risk of death and exacerbations in patients with chronic obstructive pulmonary disease: an observational cohort study of 22053 patients. Clin Microbiol Infect. 2020;26 (2):227-234.

88. Kang R, Zeng L, Xie Y, et al. A novel PINK1- and PARK2-dependent protective neuroimmune pathway in lethal sepsis. Autophagy. 2016;12(12):2374-2385.

89. Piquereau J, Godin R, Deschênes S, et al. Protective role of PARK2/Parkin in sepsis-induced cardiac contractile and mitochondrial dysfunction. Autophagy. 2013;9(11):1837-1851.

90. Yoshizumi T, Ichinohe T, Sasaki O, et al. Influenza A virus protein PB1-F2 translocates into mitochondria via Tom40 channels and impairs innate immunity. Nat Commun. 2014;5:4713.
91. Zhang X, Yuan D, Sun Q, et al. Calcium/calmodulin-dependent protein kinase regulates the PINK1/Parkin and DJ-1 pathways of mitophagy during sepsis. FASEB J. 2017;31(10):4382-4395.

92. Zhou J, Yang R, Zhang Z, et al. Mitochondrial protein PINK1 positively regulates RLR signaling. Front Immunol. 2019;10:1069.

93. Matheoud D, Cannon T, Voisin A, et al. Intestinal infection triggers parkinson's disease-like symptoms in Pink1-/- mice. Nature. 2019;571(7766):565-569.

94. Zhao C, Zhao W. TANK-binding kinase 1 as a novel therapeutic target for viral diseases. Expert Opin Ther Targets. 2019;23 (5):437-446.

95. Zachari M, Gudmundsson SR, Li Z, et al. Selective autophagy of mitochondria on a ubiquitin-endoplasmic-reticulum platform. Dev Cell. 2019;50(5):627-643.e5.

96. Richter B, Sliter DA, Herhaus L, et al. Phosphorylation of OPTN by TBK1 enhances its binding to Ub chains and promotes selective autophagy of damaged mitochondria. Proc Natl Acad Sci U S A. 2016;113(15):4039-4044.

97. Zhang Q, Kuang H, Chen C, et al. The kinase Jnk2 promotes stress-induced mitophagy by targeting the small mitochondrial form of the tumor suppressor ARF for degradation. Nat Immunol. 2015;16(5):458-466.

98. Lazarou M, Sliter DA, Kane LA, et al. The ubiquitin kinase PINK1 recruits autophagy receptors to induce mitophagy. Nature. 2015;524(7565):309-314.

99. Mohamud Y, Qu J, Xue YC, et al. CALCOCO2/NDP52 and SQSTM1/p62 differentially regulate coxsackievirus B3 propagation. Cell Death Differ. 2019;26(6):1062-1076.

100. Zhang R, Varela M, Vallentgoed W, et al. The selective autophagy receptors optineurin and p62 are both required for zebrafish host resistance to mycobacterial infection. PLoS Pathog. 2019;15(2): e1007329.

101. Shi J, Wong J, Piesik P, et al. Cleavage of sequestosome $1 / \mathrm{p} 62$ by an enteroviral protease results in disrupted selective autophagy and impaired NFKB signaling. Autophagy. 2013;9 (10):1591-1603.

102. Sin J, McIntyre L, Stotland A, et al. Coxsackievirus B escapes the infected cell in ejected mitophagosomes. J Virol. 2017;91(24): e01347-e01417.

103. Du Y, Duan T, Feng Y, et al. LRRC25 inhibits type I IFN signaling by targeting ISG15-associated RIG-I for autophagic degradation. EMBO J. 2018;37(3):351-366.

104. Xian H, Yang S, Jin S, et al. LRRC59 modulates type I interferon signaling by restraining the SQSTM1/p62-mediated autophagic degradation of pattern recognition receptor DDX58/RIG-I. Autophagy. 2020;16(3):408-418.

105. Tschurtschenthaler M, Adolph TE. The selective autophagy receptor optineurin in crohn's disease. Front Immunol. 2018;9:766.

106. Chew TS, O'Shea NR, Sewell GW, et al. Optineurin deficiency in mice contributes to impaired cytokine secretion and neutrophil recruitment in bacteria-driven colitis. Dis Model Mech. 2015;8 (8):817-829.

107. Zhang Y, Yao Y, Qiu X, et al. Listeria hijacks host mitophagy through a novel mitophagy receptor to evade killing. Nat Immunol. 2019;20(4):433-446. 


\section{Publish your work in this journal}

The International Journal of COPD is an international, peer-reviewed journal of therapeutics and pharmacology focusing on concise rapid reporting of clinical studies and reviews in COPD. Special focus is given to the pathophysiological processes underlying the disease, intervention programs, patient focused education, and self management

protocols. This journal is indexed on PubMed Central, MedLine and CAS. The manuscript management system is completely online and includes a very quick and fair peer-review system, which is all easy to use. Visit http://www.dovepress.com/testimonials.php to read real quotes from published authors.

Submit your manuscript here: https:/www.dovepress.com/international-journal-of-chronic-obstructive-pulmonary-disease-journal 